\title{
Melhoramento do Perfil Lipídico da Salsicha tipo Viena
}

\author{
Vitor C. Meleiro; Natália F. de Medeiros \& Samer Pereira
}

Para execução do trabalho, foram elaboradas três formulações de salsicha: padrão, Fonte de $\omega-3$ e alto conteúdo de $\omega-3$ e, submetidas a análises físico-química, microbiológica, sensorial e posterior avaliação estatística dos resultados por meio do método Anova. Todas as formulações atenderam aos padrões legais estabelecidos, tanto físico-químicos como microbiológicos. Na avaliação estatística, verificou-se a formação de dois grupos estatísticos diferentes, em nível de 5\%, entre a amostra padrão e as amostras com melhoramento do perfil lipídico, os resultados das avaliações sensoriais de aceitação com provadores, apresentaram boa resposta em comparação com parâmetros gerais de aprovação.

Palavras chave: salsicha; ômega 3; perfil lipídico.

For execution of work were prepared three sausage formulations: Standard, $\omega-3$ source and high $\omega-3$ content, and subjected to physical and chemical analysis, microbiological, sensory and subsequent statistical evaluation of the results through the Anova method. All formulations met the legal standards set both physicochemical and microbiological. Statistical evaluation showed the formation of two different statistical groups at the 5\% level, between the standard sample and the samples with improvement of the lipid profile, the results of sensory evaluation of acceptance with tasters showed good response compared with parameters general approval.

Key words: sausage; omega 3; lipid profile. 


\section{Introdução}

Em razão de modificações sociais, econômicas e culturais que vêm ocorrendo, nas últimas décadas, no País, foram percebidas alterações nos hábitos e comportamentos alimentares; por exemplo, o incremento da participação do consumo alimentar fora de casa, ${ }^{1}$ o aumento do consumo de alimentos processados ${ }^{2}$ e a substituição das refeições e preparações tradicionais por lanches com elevada concentração de energia, gorduras, açúcar de adição e sódio. ${ }^{3}$ Tais características desta dieta associam-se a condições relacionadas com a nutrição e o metabolismo, como a obesidade, as doenças cardiovasculares, a hipertensão, o diabetes e o câncer, ${ }^{4}$ as quais são as principais responsáveis pelos óbitos no Brasil. $^{5}$

Contribuindo com esse quadro, o aumento do poder aquisitivo da população brasileira, aumentou também o consumo de carnes que, atualmente, é o segundo produto que o consumidor brasileiro mais gastou dinheiro, logo após o pão. Dentre os produtos cárneos mais vendidos, um dos que se destaca é a salsicha. Esse aumento tem ocorrido desde a alta no consumo de cachorro-quente, que sendo vendido em bares e lanchonetes e comércio ambulante. Outro fator que contribui para o aumento no consumo deste embutido está relacionado à variedade de pratos e preparos que têm a a salsicha como base, além do baixo custo deste embutido. ${ }^{6}$

Verificando esta nova tendência de dieta, composta por alimentos processados de fácil preparo em detrimento as refeições tradicionais e, buscando colaborar para a melhoria da qualidade nutricional, buscou-se a alternativa de melhoramento do perfil de lipídios que compõem a salsicha por meio da substituição da gordura animal pelo óleo vegetal e a incorporação de um ácido graxo, com funcionalidade comprovada, objetivando oferecer um alimento de melhor qualidade nutricional e mais um fonte de ingestão do ômega $3(\omega-3)$.

\section{Métodos}

Utilizando as instalações do SENAI-RJ - CTS Alimentos e Bebidas, foram elaboradas três formulações de salsicha tipo Viena sendo; a formulação 1 padrão, em acordo com o padrão legal ${ }^{7}$, e as formulações 2 e 3 com substituição parcial de gordura animal por óleo de soja e adição do ácido graxo ômega 3 ( $\omega-3)$, em quantidades suficientes que as classifique, respectivamente, como fonte de $\omega-3$ e alto conteúdo de $\omega-3$, de acordo com o determinação legal estabelecida8. Conforme formulação na tabela 1 :

Tabela 1: formulações de salsicha tipo Viena

\begin{tabular}{|l|c|c|c|}
\hline & Padrão & Fonte & $\begin{array}{c}\text { Alto } \\
\text { Conteúdo }\end{array}$ \\
\hline Ingredientes & $\%$ & $\%$ & $\%$ \\
\hline Paleta Suína & 30,00 & 30,00 & 30,00 \\
\hline Paleta Bovina & 24,80 & 24,80 & 24,80 \\
\hline Toucinho & 20,00 & 3,00 & 3,00 \\
\hline Óleo de Soja & --- & 16,84 & 16,80 \\
\hline Ômega 3 & --- & 0,06 & 0,10 \\
\hline Gelo & 10,00 & 10,00 & 10,00 \\
\hline $\begin{array}{l}\text { Proteína Texturizada de } \\
\text { Soja (Hidratada) }\end{array}$ & 10,00 & 10,00 & 10,00 \\
\hline Fécula de Mandioca & 2,00 & 2,00 & 2,00 \\
\hline Sal & 1,80 & 1,80 & 1,80 \\
\hline Sal de Cura & 0,25 & 0,25 & 0,25 \\
\hline Fosfato & 0,30 & 0,30 & 0,30 \\
\hline Erirtobato & 0,25 & 0,25 & 0,25 \\
\hline Aroma de Alho & 0,10 & 0,10 & 0,10 \\
\hline Aroma de Fumaça & 0,10 & 0,10 & 0,10 \\
\hline $\begin{array}{l}\text { Condimentos para } \\
\text { Salsicha }\end{array}$ & 0,40 & 0,50 & 0,50 \\
\hline Total & 100,00 & 100,00 & 100,00 \\
\hline
\end{tabular}

Para avaliação da composição centesimal das três formulações, foram realizadas análises físico-químicas para determinação de proteína, gordura, umidade e cinzas em triplica para cada formulação, seguindo a metodologia estabelecida pelo Ministério da Agricultura Pecuária e Abastecimento (MAPA) ${ }^{9}$ e, a quantificação de carboidratos totais foi realizada por cálculo de diferença: Carboidratos Totais $=(\% \mathrm{P}+\% \mathrm{G}+\% \mathrm{U}+\% \mathrm{Cz})-100$

Para validação da qualidade microbiológica das formulaçõese, posterior avalição sensorial, as formulações foram submetidas a análises microbiológicas, seguindo a metodologia estabelecida Ministério da Agricultura Pecuária e Abastecimento (MAPA) ${ }^{10}$. 
Para avaliação sensorial, as formulações foram submetidas ao teste de aceitação por escala hedônica ${ }^{11}$, utilizando uma ficha de avalição, conforme figura 1. Esta avalição foi realizada em momentos distintos para cada formulação, com a intenção de evitar a comparação direta entre amostras.

Gênero: ( ) F ( ) M Data: _/_/_

Você está recebendo uma amostra de salsicha, marque a opção que melhor representa a sua opinião sobre este produto.

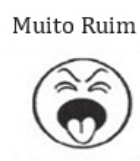

01

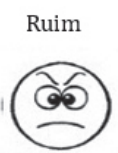

02

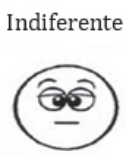

03

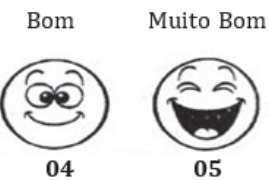

Comentários:

Figura 1: Ficha de análise sensorial

$\mathrm{Na}$ avaliação estatística, foram utilizados os escores da ficha de avaliação sensorial, com o objetivo de atribuir valores ao resultado obtido e, possibilitar quantificar percentual de aceitação para cada amostra e comparação entre as médias das diferentes amostra, por meio da Anova, para verificação se há diferença significativa, em nível de 5\%, entre as três amostras, com auxílio do programa estatístico BioEstat 5.0.

\section{Resultados}

As análises físico-químicas foram realizadas em triplicata, sem repetição, com objetivo de determinar a composição centesimal da salsicha tipo Viena e, possibilitar a comparação com o padrão legal ${ }^{12}$, estabelecido pela Instrução Normativa (IN) $\mathrm{n}^{\circ} 4$ do Ministério de agricultura e Pecuária e Abastecimento (MAPA), que aprova o Regulamento Técnico de identidade e Qualidade de salsicha.

Os resultados obtidos mostram que, em comparação com a IN n ${ }^{\circ} 4$, tanto a formulação padrão quanto as duas formulações com adição de $\omega$-3 e substituição parcial da gordura suína por óleo de soja, atendem ao padrão legal em todos os quesitos, conforme apresentado na tabela 2 .
Tabela 2: Resultados físico-químicos e comparação com padrão legal $\left(\mathrm{IN} \mathrm{n}^{\circ} 4\right)$.

\begin{tabular}{|c|c|c|c|c|}
\hline \multirow{2}{*}{ Análises } & \multicolumn{3}{|c|}{ Média \pm Desvio Padrão (\%) } & \\
\cline { 2 - 5 } & Padrão & Fonte & $\begin{array}{c}\text { Alto } \\
\text { Conteúdo }\end{array}$ & IN n 4 \\
\hline \multirow{2}{*}{ Umidade } & $61,06 \pm$ & $56,81 \pm$ & $55,21 \pm$ & $65 \%$ \\
& 0,44 & 0,06 & 0,33 & máx. \\
\hline \multirow{2}{*}{ Gorduras } & $11,97 \pm$ & $18,67 \pm$ & $23,50 \pm$ & $30 \%$ \\
& 0,92 & 0,12 & 0,41 & máx. \\
\hline \multirow{2}{*}{ Proteínas } & $16,51 \pm$ & $16,66 \pm$ & $14,66 \pm$ & $12 \%$ \\
& 0,18 & 0,00 & 0,54 & mín. \\
\hline \multirow{2}{*}{ Carboidratos } & $9,44 \pm$ & $5,09 \pm$ & $3,78 \pm$ & $7 \%$ \\
& 1,26 & 0,31 & 0,77 & máx. \\
\hline \multirow{2}{*}{ Cinzas } & $1,07 \pm$ & $2,77 \pm$ & $2,84 \pm$ & - \\
& 0,03 & 0,20 & 0,20 & - \\
\hline
\end{tabular}

$\mathrm{Na}$ comparação entre a formulação padrão e as formulações com alteração do perfil lipídico, percebe-se que, como as formulações como a modificação no perfil lipídico atendem aos parâmetros de composição físicoquímica da salsicha, estas contribuem para a melhoria das características nutricionais deste produto, visto que, colabora com a redução na ingestão de gorduras saturas e incorpora $\omega$-3 à composição.

A avaliação microbiológica teve por princípio a validação das condições higiênico-sanitárias do processo e do produto para, posteriormente, submetê-lo à avaliação sensorial comprovadores. Para comprovação destes parâmetros, após o processamento todas as formulações ,foram submetidas a análises microbiológica, tendo como parâmetros de comparação o padrão legal estabelecido pela RDC ${ }^{12}$ do Ministério da Saúde ${ }^{13}$. Estão dispostos, na tabela 3 , os resultados das análises microbiológicas para as três formulações, seguido do padrão estabelecido pela RDC ${ }^{12}$.

Tabela 3: Resultados microbiológicos e comparação com padrão legal (RDC12)

\begin{tabular}{|c|c|c|c|c|}
\hline $\begin{array}{c}\text { Micro- } \\
\text { organismo }\end{array}$ & Padrão & Fonte & $\begin{array}{c}\text { Alto } \\
\text { Conteúdo }\end{array}$ & RDC 12 \\
\hline $\begin{array}{c}\text { Coliformes a } \\
45^{\circ} \mathrm{C} / \mathrm{g}\end{array}$ & $\begin{array}{c}7 \\
\mathrm{NMP} / \mathrm{g}\end{array}$ & $\begin{array}{c}4 \\
\mathrm{NMP} / \mathrm{g}\end{array}$ & $4 \mathrm{NMP} / \mathrm{g}$ & $\begin{array}{c}<103 \\
\mathrm{NMP} / \mathrm{g}\end{array}$ \\
\hline $\begin{array}{c}\text { S. coagulase } \\
\text { positiva }\end{array}$ & $\begin{array}{c}12 \times 102 \\
\mathrm{UFC}\end{array}$ & $\begin{array}{c}14 \times 102 \\
\mathrm{UFC}\end{array}$ & $\begin{array}{c}12 \times 102 \\
\mathrm{UFC}\end{array}$ & $\begin{array}{c}<3 \times 103 \\
\mathrm{UFC}\end{array}$ \\
\hline $\begin{array}{c}\text { C. sulfito } \\
\text { redutor }\end{array}$ & $5,9 \times 10$ & $3,7 \times 10$ & $3,1 \times 10$ & $\begin{array}{c}<3 \times 102 \\
\mathrm{UFC}\end{array}$ \\
\hline Salmonella sp. & $\begin{array}{c}\text { Aus. } / \\
25 \mathrm{~g}\end{array}$ & $\begin{array}{c}\text { Aus. } \\
/ 25 \mathrm{~g}\end{array}$ & Aus. $/ 25 \mathrm{~g}$ & $\begin{array}{c}\text { Ausência } \\
/ 25 \mathrm{~g}\end{array}$ \\
\hline
\end{tabular}


Os resultados microbiológicos mostraram que, em comparação com a $\mathrm{RDC}^{12}$, o processo e os produtos elaborados atendem aos padrões legais e, desta forma, encontram-se me acordo para realização dos testes sensoriais com provadores.

Foi realizado o teste de aceitação por escala hedônica, em que cada julgador opinou o quanto gostou ou não de cada uma das três formulações. Para tanto, foi utilizado um universo de 136 julgadores, entre alunos e professores do CTS-Alimentos e Bebidas - SENAI Vassouras - RJ, conforme exposto na figura 2.

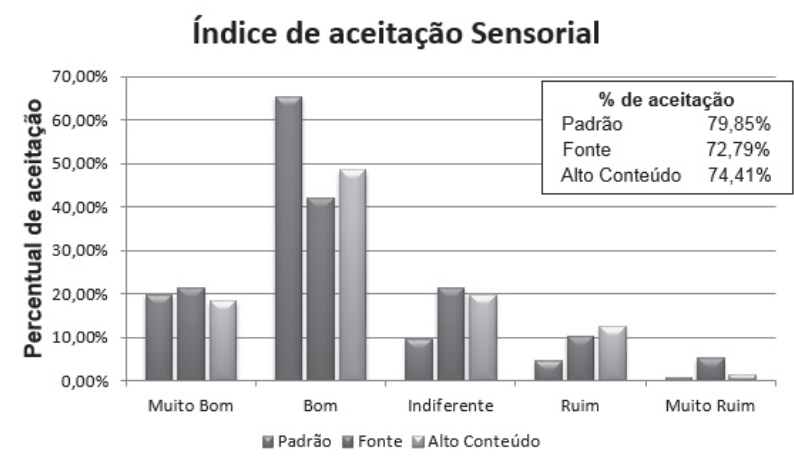

Figura 2: Resultados da análise sensorial de salsicha

Os resultados da análise sensorial mostraram que a formulação padrão obteve um índice de aceitação $(79,85 \%)$ mais alto que as formulações fonte de $\omega-3$ $(72,79 \%)$ e alto conteúdo de $\omega-3 \quad(74,41 \%)$. Ainda assim, pode-se afirmar que os índices de aceitação são satisfatórios tanto para a formulação padrão quanto para as formulações com adição de $\omega-3$, já que, um produto para ser considerado aceito é necessário que o resultado da analise sensorial seja maior que $70 \%{ }^{14}$.

No tratamento estatístico, foi aplicado o teste de Anova de um critério, para comparação entre as médias de aceitação obtidas na análise sensorial e, verificação se há diferença significativa, em nível de 5\%, para a aceitação das amostras analisadas.

Nos resultados descritos na tabela 4, percebe-se que o valor de $\mathrm{F}(5,60254)$ é maior que o valor de $\mathrm{F}$ crítico $(3,01800)$ e o valor $\mathrm{P}(0,00398)$ é menor que 0,05 . Assim, estes parâmetros mostram que, existe diferença significativa, em nível de 5\%, para as médias obtidas em análise sensorial entre as amostras de salsicha.
Tabela 4: Resultado de Anova com médias sensoriais

\begin{tabular}{|c|c|c|c|c|c|c|}
\hline $\begin{array}{c}\text { Fonte da } \\
\text { variação }\end{array}$ & SQ & gl & MQ & F & valor-P & F crítico \\
\hline $\begin{array}{c}\text { Entre } \\
\text { grupos }\end{array}$ & 9,55425 & 2 & 4,94362 & 5,60254 & 0,00398 & 3,01800 \\
\hline $\begin{array}{c}\text { Dentro } \\
\text { dos } \\
\text { grupos }\end{array}$ & 357,367 & 405 & 0,88238 & & & \\
\hline Total & 367,254 & 407 & & & & \\
\hline
\end{tabular}

Para determinar a diferença entre as médias de aceitação das amostras de salsicha, foi aplicado o teste de Tukey, como descrito na tabela 5.

Tabela 5: Resultado para o teste de Tukey e agrupamento das amostras

\begin{tabular}{|l|c|c|}
\hline & $\begin{array}{c}\text { Média } \pm \text { Desvio } \\
\text { Padrão }\end{array}$ & DMS \\
\hline Padrão & $3,99 \pm 0,73 \mathrm{a}$ & 0,29 \\
\hline Fonte & $3,64 \pm 1,08 \mathrm{~b}$ & 0,29 \\
\hline Alto Conteúdo & $3,69 \pm 0,96 \mathrm{~b}$ & 0,29 \\
\hline
\end{tabular}

A diferença obtida entre as médias de aceitação das amostras de salsicha $(0,29)$ separa as amostras em dois grupos, a e b, em que a amostra de melhor média de aceitação foi a Padrão, configurando o grupo a e as amostras com melhoramento do perfil lipídio formam um segundo grupo b, em que, entre estas duas amostras, não há diferença significativa, em nível de $5 \%$, para análise sensorial de aceitação.

\section{Conclusão}

O melhoramento da qualidade nutricional da salsicha, por meio da substituição de gordura animal por óleo de soja e incorporação do ácido graxo $\omega-3$, mostrou-se totalmente viável dos pontos de vista tecnológico, legal e sensorial, tendo em vista que, a tecnologia aplicada para elaboração da salsicha com melhoramento do perfil lipídico foi a mesma utilizada na fabricação da salsicha padrão sem necessidades de adequações nos equipamentos ou processo. Do ponto de vista legal, as análises de composição físico-química mostraram 
que todas as amostras estão em conformidades com os parâmetros legais estabelecidos. Na avaliação sensorial, observou-se que as amostras padrão, fonte de $\omega$-3 e alto conteúdo de $\omega-3$, obtiveram índice de aceitação maior que $70 \%$ e, mesmo considerando que houve diferença significativa, em nível de 5\%, entre a amostra padrão e as amostras com melhoramento do perfil lipídico, todas superam o valor mínimo para que um produto seja considerado aceito.

\section{Referências}

1. Bezerra IN, Sichieri R. Eating out of homeand obesity: a Brazilian nationwide survey.Public Health Nutr.2009;12(11):2037-43. DOI: 10.1017 / S 1368980009005710.

2. Dishchekenian VRM, Escrivão MAMS, Palma D,AnconaLopes F, Araújo EAC, Taddei JAAC. Padrões alimentares de adolescentes obesos e diferentes repercussões metabólicas. Rev Nutr. 2011;24(1):17-29.DOI:10.1590/S141552732011000100002 .

3. Monteiro CA, Levy RB, Claro RM, Castro IR, Cannon G. Increasing consumption of ultra-processed foods and likely impact on human health: evidence from Brazil. Public Health Nutr. 2011;14(1):5-13. DOI:10.1017/S1368980010003241.

4. Malik VS, Poppkin BM, Bray GA, Despres JP, Willett WC, $\mathrm{Hu}$ FB. Sugar-sweetened beverages and risk of metabolic syndrome and type 2 diabetes: a metaanalysis. Diabetes Care. 2010;33(11):2477-83. DOI:10.2337/dc10-1079.

5. Schmidt MI, Duncan BB, Silva GA, Menezes AM, Monteiro CA, Barreto SM, et al. Chronic noncommunicable diseases in Brazil: burden and current challenges. Lancet. 2011;377(9781):1949-61. DOI:10.1016/S0140-6736(11)60135-9.

6. IBGE, Instituto Brasileiro de Geografia e Estatística www.ibge. gov.br; acessado 10/11/2013.

7. Brasil. Ministério da Agricultura Pecuária e Abastecimento MAPA. Instrução Normativa - 4, DE 31/03/2000. Regulamentos Técnicos de identidade qualidade de carne mecanicamente separada, salsicha mortadela e linguiça.

8. Brasil.. Agência Nacional de Vigilância Sanitária - Anvisa. RDC - 54, de 12/11/2012 Regulamento Técnico Mercosul sobre Informação Nutricional Complementar.

9. Brasil.. Ministério da Agricultura Pecuária e Abastecimento - MAPA Instrução Normativa n. 20 de 21/07/99. Métodos Analíticos Físico-químicos para Controle de Produtos Cárneos e seus Ingredientes.

10. Brasil.. Ministério da Agricultura Pecuária e Abastecimento - MAPA Instrução Normativa n. 62 de 26/08/2003 Métodos Analíticos Oficiais para Análises Microbiológicas para Controle de produtos de Origem Animal e Água.
11. Minim, V. P. R. Análise Sensorial: estudo com consumidores. Viçosa, MG: Ed. UFV, 2006. 225 p.

12. Brasil.. Instrução Normativa - 4, DE 31/03/2000. Regulamentos Técnicos de identidade qualidade de carne mecanicamente separada, salsicha mortadela e linguiça.

13. Brasil.. Agência Nacional de Vigilância Sanitária - Anvisa ${ }^{\circ} 12$ de 02 de janeiro de 2001. Regulamento Técnico sobre padrões microbiológicos para alimentos.

14. Teixeira, e.; Meinert, E. M.; \& Barbetta, P. A. Análise Sensorial de Alimentos. Série Didática. Florianópolis: Editora UFSC, 1987, p $18-102$

\section{Vitor C. Meleiro*; Natália F. de Medeiros \& Samer Pereira}

Centro de Tecnologia SENAI Alimentos e Bebidas. Rua Nilo Peçanha, 85, Centro, Vassouras, Rio de Janeiro, Brasil. CEP 27.700-000.

*E-mail: vmeleiro@firjan.org.br 